\title{
IN-VITRO INVESTIGATIONS ON THE INFLUENCE OF ANGIOPLASTY ON THE MECHANICAL PROPERTIES OF ATHEROSCLEROTIC ARTERIES.
}

\author{
Jacco L.G. Steenhuijsen*, Simon R. Vaartjes ${ }^{+}$, Cees J. Poortermans ${ }^{+}$, Herman B.K. Boom* \\ * Institute for BioMedical Technology, University of Twente, P.O.Box 217, $7500 \mathrm{AE}$ \\ Enschede, The Netherlands \\ $+\quad$ Hospital Medisch Spectrum Twente, Enschede, The Netherlands
}

\begin{abstract}
Knowledge of the influence of Percutaneous Transluminal (Coronary) Angioplasty on the mechanical properties of atherosclerotic arteries is scarce. We performed experiments to determine the elasticity, viscosity and minimal diameter of arteries before and after angioplasty. Our results show significant changes in elasticity and viscosity of arterial segments, but show that minimal arterial diameter remains unchanged. This suggests that the effect of angioplasty is not primarily caused by change in arterial diameter, but is generated by a change in arterial elasticity as well as viscosity.
\end{abstract}

Keywords: angioplasty, mechanical parameters, elasticity, viscosity

\section{Introduction}

Knowledge of the influence of Percutaneous Transluminal (Coronary) Angioplasty (PT(C)A) on the mechanical properties (elasticity, viscosity) of atherosclerotic arteries is scarce. In most of the investigations, attention is paid to the change in diameter of the lumen. Most studies define 'Successful angioplasty' in terms of enlargement of minimal arterial diameter rather than using a definition based on hemodynamic properties of the artery (e.g. increase of bloodflow).

Recently Botas[1], compared segmental coronary distensibility before and after PTCA. He concluded that most of the enlargement of the diameter at physiologic pressure can be subscribed to increased distensibility after angioplasty. This increased distensibility should result in passive distention of the vessel in response of the blood pressure. Botas, however, made use of an angioplasty balloon inflated up to 5 bar to measure arterial distensibility. In this way, the artery is not free to change shape as a result of increased pressure. Furthermore, no attention was paid on the viscous properties of the arterial wall.

The study we describe here was conducted to evaluate the effects of balloon-inflation on elasticity, viscosity and minimal arterial diameter of the arterial wall at physiologic pressures.

\section{Methods}

Human femoral arteries were excised during autopsy after informed consent and cleaned from fat and other tissue. The arteries were kept in glucose-free Tyrodesolution at $4^{\circ} \mathrm{C}$ until the experiment was performed.
Before the experiment, arteries were fixed at physiologic length in a bath filled with Tyrode-solution with glucose. The solution was oxygenated with a mixture consisting of $5 \% \mathrm{O}_{2}$ en $95 \% \mathrm{CO}_{2}$. Temperature and $\mathrm{pH}$ of the solution were kept at physiological values $[2,3]$.

Before the experiment was performed, mean inner and outer diameter of the artery as well as the area of arterial-wall were measured by ultrasound (Diasonics Master, $10 \mathrm{MHz}$ linear array, resolution $0.15 \mathrm{~mm}$, reproducibility $\pm 0.2 \mathrm{~mm})$. A tank filled with Tyrode-solution was connected to the lumen of the arterial segment. Intraluminal pressure was changed by moving the tank up and down. Intraluminal pressure could be changed this way in any (continuous) pressure-time relation we wanted. For these experiments we used sinusoidal pressure-time relations. Intraluminal pressure was measured using a Spectramed DTXPlus pressure-transducer between the artery and the tank. Arterial dimensions were measured using 4 Linear Variable Differential Transducers (Jensen LDT-51, reproducibility of mean arterial diameter $0.012 \mathrm{~mm}$ ). The mean arterial diameter was calculated from two orthogonal diameters to exclude effects of possible translations of the artery during the experiment.

After measurement of the mechanical properties of the artery, an angioplasty ballooncatheter (Olbert, Meadox Surgimed A/S) was inflated inside the artery. The balloon was inflated by a computer-controlled system until maximum balloon-pressure was reached or until first signs of arterial damage were discovered[3].

After the balloon-inflation, the mechanical properties of the artery were measured again, using the same procedure and parameters as before the balloon-inflation.

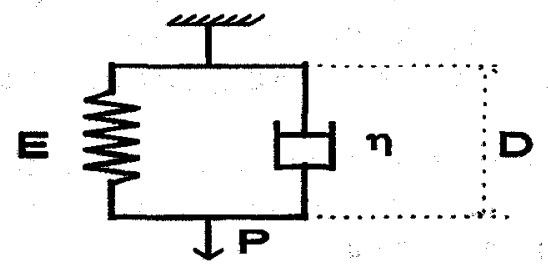

Figure 1: Representation of the Voigt-model, consisting of a spring with elasticity $E$ and a dashpot with viscosity $\eta$ in parallel. 

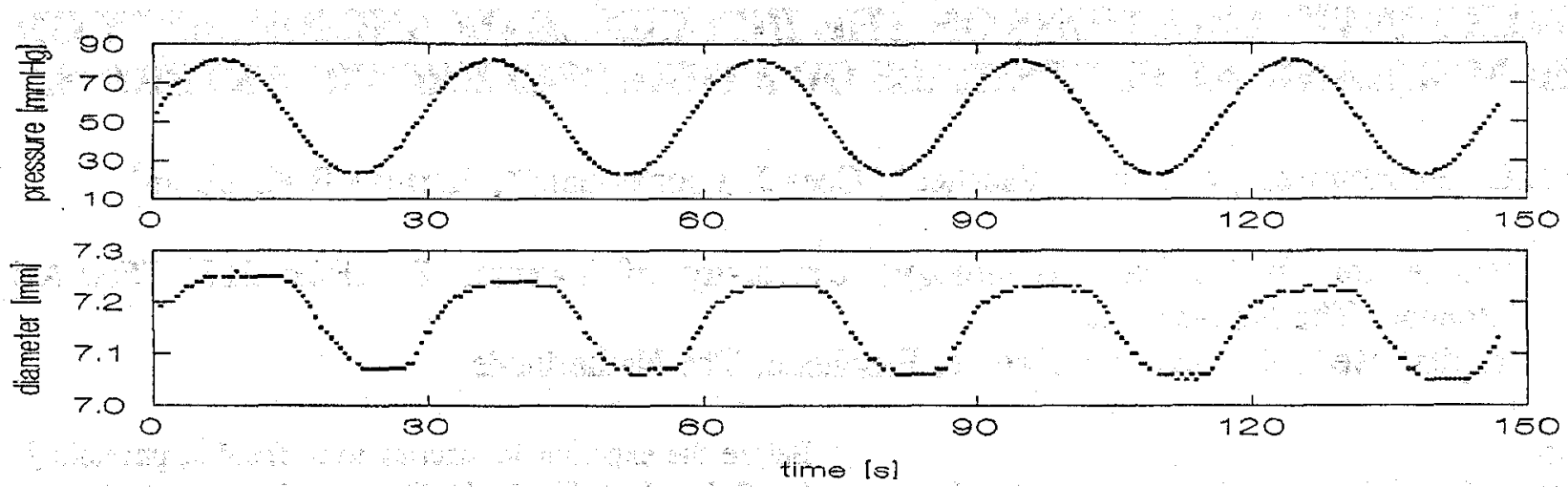

Figure 2: Plot of the pressure-time relation used in our experiments and a resulting diameter-time curve.

Definition of mechanical parameters

We used a mechanical model consisting of a spring with elasticity $E$ and a dashpot with viscosity $\eta$ in parallel (Voigt model, figure 1) to describe the relationship between the intraluminal pressure $P$ and the outer diameter of the artery $D$. Applying a sinusoidal pressure-time relationship $P(t)=P_{0}+P_{1}{ }^{*} \sin (\omega t)$ to this model, will lead to a sinusoidal change in diameter $D(t)=D_{0}+D_{1}{ }^{*} \sin (\omega t-\varphi)$. The parameters $E$ and $\eta$ can be derived by using the following equations in combination with the results of our measurements:

$$
\begin{aligned}
& E=\left(\frac{P_{1}}{D_{1}}\right) * \frac{1}{\sqrt{1+\tan ^{2} \varphi}} \\
& \eta=\frac{E \tan \varphi}{\omega}
\end{aligned}
$$

The minimal arterial diameter $D_{\min }$ is defined as $D_{\min }=D_{0}-D_{1}$.

\section{Results}

We measured the mechanical properties of 9 atherosclerotic arteries just before and after balloonangioplasty The period of the sinusoidal pressure-time variations we used was 30 seconds. Figure 2 shows pressure-time and diameter-time curves as measured in one of our experiments.

Values for $P_{1}, D_{1}$ and $\varphi$ determined by curve-fitting the experimental data using teh statistical package SYSTAT. Values for $E$ and $\eta$ are obtained using formula (1) and (2).

Our results show significant changes in arterial elasticity $(|\Delta \mathrm{E}|=33 \pm 18 \%)$ and changes in arterial viscosity $(|\Delta \eta|=42 \pm 39 \%)$ as a result of dilatation. Minimal arterial diameter remained unchanged $(0 \pm 9 \%)$.

The results can be divided in two groups: 3 experiments showed decreased elasticity $(\Delta E=-25 \pm 19 \%)$ and decreased viscosity $(\Delta \eta=-17 \pm 9 \%) ; 6$ experiments showed increased elasticity $(\Delta E=38 \pm 19 \%)$ as well as increased viscosity $(\Delta \eta=54 \pm 43 \%)$.

Both groups showed unchanged minimal arterial diameter $\left(\Delta D_{\min }=5.6 \pm 8 \%(\mathrm{n}=3), \Delta D_{\min }=2.4 \pm 9 \%(\mathrm{n}=6)\right)$.

\section{Conclusion and discussion}

These results show that balloon-angioplasty significantly changes arterial elasticity and viscosity, but the do not show changes in minimal arterial diameter. This suggests that the effect of angioplasty is not primarily caused by change in arterial diameter, but is generated by a change in arterial elasticity as well as viscosity. It's widely accepted that the mechanism of angioplasty is based on inducing ruptures in the plaque and the arterial wall. It is to be expected that damaging structures inside the artery will lead to a decreased elasticity. Our experiments show that changes in elasticity and viscosity of the arterial wall are important consequences of angioplasty. However, in some cases arterial elasticity appeared to have been increased by the dilatation. We conclude that the mechanical mechanism of angioplasty is not yet understood completely an that further research is needed to understand the relation between the mechanical consequences of angioplasty and the success after the procedure.

\section{Acknowledgements}

We want to thank the Netherlands Heart Foundation for supporting this research (grant 92-399) and Meadox Surgimed A/S for providing the angioplasty balloons.

\section{References}

[1] J.Botas, D.A.Clark, F.Pinto, A.Chenzbraun, T.A.Fischell, "Balloon angioplasty results" in increased segmental coronary distensibility: a likely mechanism of percutaneous transluminal coronary angioplasty", J.Am.Coll.Cardiol 1994;23;1043-52

[2] S.R.Vaartjes, J.C.Poortermans and M.J.Botman, "Invitro study of the biomechanics of atherosclerotic human arteries during angioplasty", abstracts of the 2nd European Conference on engineering and medicine, Stuttgart, Germany, april 25-28,1993,127-8

[3] J.L.G.Steenhuijsen, S.R.Vaartjes, C.J.Poortermans, M.G. Havenith and H.B.K.Boom, "A method to detect rupture of the arterial wall during balloon angioplasty", abstracts of the 16th conference of the IEEE EMBS, November 3-6,1994, Baltimore, Maryland, 860-861 